\title{
Preface
}

\section{Evolving Neuroanesthesia Practice}

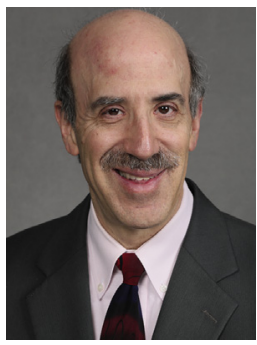

Jeffrey R. Kirsch, MD, FASA

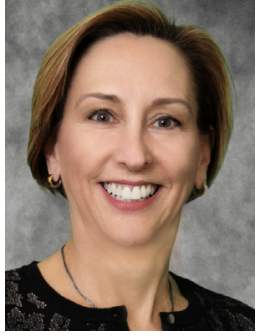

Cynthia A. Lien, MD

Editors

The goal of writing this issue was to provide an up-to-date management approach to the patient with neurologic disease that is amenable to treatment through surgical or intravascular intervention. It is critical that the anesthesia care provider stay current on evolving surgical and interventional approaches to the management of patients with neurologic disease. Those diseases that we considered most important to address in this issue were tumors, trauma, and congenital problems in the cranium and in the spine. We were pleased to be able to recruit expert authors who practice at some of the busiest academic medical centers in the world.

For neurovascular disease, we have included articles to address anesthetic management for both open and intravascular approaches. While not covered in this issue, over the past several months the impact of a COVID-19 infection on neurologic health has been recognized and needs to be mentioned. Unfortunately, COVID-19 significantly increases the rate of stroke in infected individuals with no history of vascular disease. This is a relatively new disease, and we are still learning about it, but it appears that infected patients may have a greater need for anesthesia and intravascular intervention.

There are separate articles to address the anesthetic management of intracranial and spinal cord tumors and trauma to the spinal cord and the head. Although these articles focus primarily on diseases of adult patients, the concepts are generally transferable to pediatric patients. There is one article that focuses on a primarily pediatric topic, as it relates to the anesthetic management for children with craniosynostosis.

The current practices discussed in these articles reflect current management, and they are evolving as our understanding of the pathophysiology of neurologic disease increases. That being said, there is a desperate need for additional research to improve the anesthesia care in patients with neurologic disease. The editors and authors have decided to donate all the royalties related to the sale of this issue to the Foundation for 
Anesthesia Education and Research to support the studies that will further improve our care of patients with these disease entities.

\section{DEDICATION}

Jeffrey R. Kirsch, MD, FASA: I dedicate this issue to my wife, Robin, for her unwavering love and support, and to my grandchildren, Noa, Romi, Mika, and Daniel Reese, who are my shining stars.

Cynthia A. Lien, MD: For my husband, Dan, and our two daughters, Erika and Christina, for their steadfast encouragement and understanding.

Jeffrey R. Kirsch, MD, FASA

Department of Anesthesiology

Medical College of Wisconsin

8700 W Wisconsin Avenue

Anesthesiology, 3rd Floor

Administrative Suite

Milwaukee, WI 53226, USA

Cynthia A. Lien, MD

Department of Anesthesiology Medical College of Wisconsin

8700 W Wisconsin Avenue Anesthesiology, 3rd Floor Administrative Suite

Milwaukee, WI 53226, USA

E-mail addresses:

jekirsch@mcw.edu (J.R. Kirsch)

clien@mcw.edu (C.A. Lien) 